\title{
Silver/Polyaniline Nanocomposite for the Electrocatalytic Hydrazine Oxidation
}

\author{
Ravindra P. Singh $\cdot$ A. Tiwari $\cdot$ Avinash C. Pandey
}

Received: 19 April 2011/Accepted: 18 July 2011/Published online: 13 August 2011

(C) The Author(s) 2011. This article is published with open access at Springerlink.com

\begin{abstract}
Silver/polyaniline (Ag-PANi) nanocomposites were prepared via in situ reduction of silver in aniline by mild photolysis performed with short wavelength $(365 \mathrm{~nm})$ radiation from UV lamp for $12 \mathrm{~h}$. Reduction of the silver in aqueous aniline leads to the formation of silver nanoparticles which in turn catalyze oxidation of aniline into polyaniline. A slightly broadened X-ray diffraction (XRD) pattern suggests small particle which was size consistent with cubic silver nanoparticles. The UV-Vis absorption revealed that the bands at about 400-420 nm due to benzonoid ring of the polyaniline are overlapped and blueshifted due to the presence of silver nanoparticles in powdered state. Scanning electron microscopy (SEM) of the silver/polyaniline (Ag-PANi) nanocomposite showed a size distribution with nanofibers and granular morphology of silver nanoparticles. Our findings are not only the promising approach for electro-catalytic hydrazine oxidation but also utilized in the other bio-sensing applications.
\end{abstract}

Keywords Silver/polyaniline (Ag-PANi) .

Chemical synthesis · Polymer · Nanocomposite

Presented in part at the 1st International Congress on Advanced Materials held in Jinan, PRC from May 12-17, 2011.

R. P. Singh $(\bowtie) \cdot$ A. C. Pandey

Nanotechnology Application Centre, University of Allahabad, Allahabad, India

e-mail: rpsnp169@gmail.com

A. C. Pandey

e-mail: prof.acpandey@gmail.com

\section{A. Tiwari}

National Institute for Materials Science, Tsukuba 3050047 , Japan

e-mail: ashunpl@gmail.com

\section{Introduction}

The silver nanoparticles have potential in technological applications due to its interesting properties including catalysis, conductive inks, thick film pastes, and adhesives for various electronic components, in photonics and in photography [1-3]. The conducting polymers have the excellent hosts for trapping nanoparticles of metals and semiconductors, because of their ability to act as stabilizers or surface capping agents. The nanoparticles embedded or encapsulated in a polymer; the polymer terminates the growth of the particles by controlling the nucleation. For application in opto-electronics and electronics, the control of particle size and their uniform distribution within the polymer is the key to technology. Conducting organic polymers especially doped polyaniline, which have high electrical conductivity with great environmental stability in organic, inorganic, and aqueous medium. The methods of preparation may lead to small differences in physical and chemical properties of the product culminated into the electronic applications such as sensing [4]. However, the synthesis of Ag-PANi nanocomposite via photo-polymerization process has reported for the first time for the electrocatalytical hydrazine oxidation. Hydrazine is an agricultural chemicals (pesticides), blowing agents, pharmaceutical intermediates, photographic developers, treatment of water for corrosion protection, textile dyes, and jet and rocket fuels [5]. Besides these, individuals occupationally exposed to hydrazine in the workplace or from accidental discharges into water, air, and soil, which may also occur during storage, handling, transport, and improper waste disposal [6]. The photolysis of aniline into polyaniline utilizing $\mathrm{AgNO}_{3}$ is an alternate strategy for in situ polymerization of aniline was established. This method has considered more suitable for synthesis of not only 
nanoparticles but also nanocomposite. The preparations of nanoparticles of silver by photolysis are rather scarce, although a large number of chemical methods have reported. However, the synthesis of silver nanoparticles of desired shape and size with uniform distribution within the matrix remains highly challenging and meaningful.

Polyaniline-AgNPs nanocomposites show enhanced sensing, electrical and catalytic capabilities, as compared with those of pure PANi. Khanna et al. [7] have reported the synthesis of Ag-PANi nanocomposites via in situ photo-redox mechanism by which radiation from UV lamps have used to reduce silver salt in aniline. Kang et al. [8] have prepared nanocomposites by oxidative polymerization of aniline-stabilized Ag colloids by gamma-irradiation. Karim et al. [9] jave synthesized core-shell silver nanocomposites by in situ gamma radiation-induced chemical polymerization method. Du et al. [10] have employed one-pot synthesis method for Ag/polyaniline nanoparticles; whereas Pillalamarri et al. [11] have also used one-pot synthesis method in which composite materials consisting of polyaniline nanofibres decorated with noble-metal (Ag or $\mathrm{Au}$ ) nanoparticles were synthesized with $\gamma$-radiolysis. Zhou et al. [12]. have applied the unsymmetrical square wave current method, and characterized by the combination of an anodic process of aniline monomer polymerization and a cathodic process of metal ionic electrodeposition to produce the PANi silver nanocomposite film.

Although the limitations associated with these methods are very hard to avoid, like sub-micrometre size diameter, risk of contamination and complex process control. To the best of our knowledge, the PANi/silver nanocomposite films for the elctrocatalytic oxidation of hydrazine have not reported until date. The advantage of the present method requires no other template for the reduction of silver salt into AgNPs. In this manuscript, an easy route to a silver/ polyaniline nanocomposite powder was prepared using UV lamp at $365 \mathrm{~nm}$ wavelength. Silver nitrate has reduced by aniline in an aqueous medium via photolysis to produce PANi, in which silver acts as a catalyst to initiate the polymerization in aqueous medium.

\section{Experimental}

Aniline, silver nitrate and ethanol were obtained from Merck, India. Aqueous solutions were prepared using deionized water. Aniline stored in dark at low temperature in the concentration of $0.5 \mathrm{M}$ aniline mixed with $0.5 \mathrm{M}$ of silver nitrate in total volume $(5.0 \mathrm{~mL})$ of mixture. The assay mixture had irradiated using $365 \mathrm{~nm}$ wavelength for $12 \mathrm{~h}$ at room temperature in UV chamber. The colour of the solution changes from whitish to blue and then finally it becomes green.The solution kept inrefrigerator at rest for $24 \mathrm{~h}$ to complete the polymerization process and then this solution was centrifuged at $5,000 \mathrm{rpm}$ for $30 \mathrm{~min}$. The mass obtained was washed with acetone, ethanol and double distilled water to remove excess of monomer, oligomer. The powdered sample have characterized by several techniques. ITO substrates were cleaned using distilled water with several times and air-dried. The reaction mixture well vortexes and put on the desired area of the substrate. After that, put inside the UV lamp chamber $(365 \mathrm{~nm})$ carefully for $12 \mathrm{~h}$. The presence of a green thin PANi film on ITO substrate has observed, suggesting the protonation of the emeraldine base. The AgNPs/PAni film on the ITO electrode washed with buffer solution.

Morphology of nanocomposites are studied by a Scanning electron microscopy (SEM) FEI, model Quanta 200, Netherland. The optical absorption band has measured with UV-Vis spectroscopy (Perkin Elmer model L35, Germany). Photoluminescence spectra of the samples have obtained out using LS 55 Fluorescence spectrophotometer (Perkin Elmer). The excitation wavelength for the PL study of the sample is $330 \mathrm{~nm}$. TGA of the samples have carried out on Pyris 1 TGA instrument at a heating rate of $10{ }^{\circ} \mathrm{C}$ per minute in $\mathrm{N}_{2}$ atmosphere over a temperature range of $25-700{ }^{\circ} \mathrm{C}$. FTIR is recorded with a FTIR-NEXUS, NICOLET instrument in the region of $500-4000 \mathrm{~cm}^{-1}$ using $\mathrm{KBr}$ pellet. XRD was performed on Rigaku D/max$2200 \mathrm{PC}$ diffractometer operated at $40 \mathrm{kV} / 20 \mathrm{~mA}$, using $\mathrm{Cu} \mathrm{K} \alpha_{1}$ radiation with wavelength of $1.54 \AA$ in the wide angle region from 20 to $80^{\circ}$. Cyclic voltammetry $(\mathrm{CV})$ and differential pulse voltammetry (DPV) were performed on electrochemistry workstation CHI 660A (Austin, USA). Electrochemical measurements have performed in onecompartment cell containing the ITO modified electrode (geometric area of $1.0 \mathrm{~cm}^{2}$ ) as working electrode, a platinum auxiliary electrode, and $\mathrm{Ag} / \mathrm{AgCl}$ sat electrode as reference. DPV experiments have performed at a scan rate of $20 \mathrm{mVs}^{-1}$ using instrumental conditions of $50 \mathrm{mV}$ pulse amplitude, pulse width of $50 \mathrm{~ms}$ and pulse time of $200 \mathrm{~ms}$.

\section{Results and Discussions}

The excitation of aniline monomer by UV light @ 365 nm is playing role for the polyaniline formation and controlling of silver particle sizes. The speculation to this mechanism is also due to $\mathrm{Ag}^{+}$ions, which oxidize the aniline monomer and as result reduce it into metal $\mathrm{Ag}^{0}$ and finally PANi and $\mathrm{Ag}^{0}$ particles are formed. Possibly, in another way, the decomposition of $\mathrm{NO}^{3-}$ by UV light yields hydroxyl radicals $(\mathrm{OH})$ as oxidizing agent and they are responsible for aniline photopolymerization rather than rather than due to $\mathrm{Ag}^{+}$. Thus, we can propose that $\mathrm{NO}^{3-}$ ions are able to 
promote the polymerization of aniline monomer by UV light. Strongly we can state that the exact mechanism of photopolymerization process are still not fully understand, probably UV light interacts with aniline and silver nitrate to yield PANi and $\mathrm{Ag}^{0}$ due to photo-excitation process.

Figure 1 shows UV-Vis absorption spectrum of polyaniline-silver nanocomposite. The polyaniline-silver nanocomposite displays a blue shift of the absorption bands. It is well established that metal nanoparticles display surface plasmon resonance bands in the UV-Vis region due to the excitation of electrons. An additional absorption band is obtained at around $370 \mathrm{~nm}$ for the polyaniline composite and it corresponds to the surface plasmon resonance absorption band of silver nanoparticles indicating that the nanoparticles are existed in the nanocomposite.

Figure 2 shows photoluminescence (PL) of standard polyaniline (a) and polyaniline-silver nanocomposite (b). PL spectrums of standard polyaniline (sigma) and

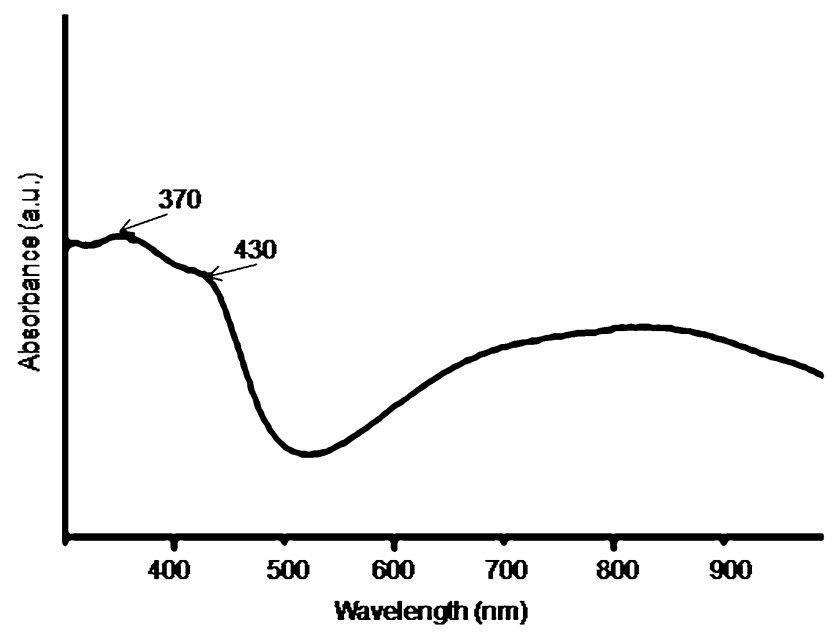

Fig. 1 Shows UV-Vis absorption spectrum

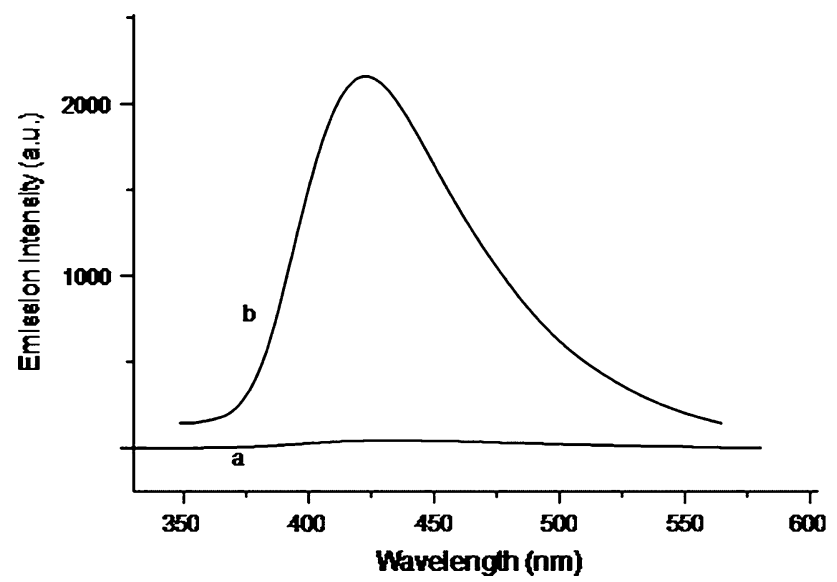

Fig. 2 Photoluminescence (PL) of standard polyaniline and polyaniline-silver nanocomposite polyaniline-silver nanocomposite as prepared are observed and suggested the intensity of PL increases with content of silver nanoparticles. The emission peak is centered at $443 \mathrm{~nm}$. In this investigation exciton wavelength is taken as $330 \mathrm{~nm}$ to excite silver nanoparticles may be due to the $\pi^{*}$ transition of the benzenoid unit of polyaniline.

Figure 3 shows the FTIR spectrum of standard polyaniline (a) and polyaniline-silver nanocomposite (b). In corporation of silver nanoparticles in polyaniline matrix leads to small shift of the peaks to the lower wavelengths and also decreases in the intensity of peaks, which indicates that the structural change of polymer occurs. The infrared spectra of the nanocomposite films are similar to the standard PANi, confirming the formation of PANi. There is a shift in the peaks associated with $\mathrm{C}=\mathrm{C}$ and $\mathrm{C}=\mathrm{N}$ stretching of the quinoid ring and $\mathrm{C}-\mathrm{H}$ bending of the benzene ring.

Figure 4 shows XRD pattern of standard polyaniline (a), AgNPs (b) and polyaniline-silver nanocomposite (c). The

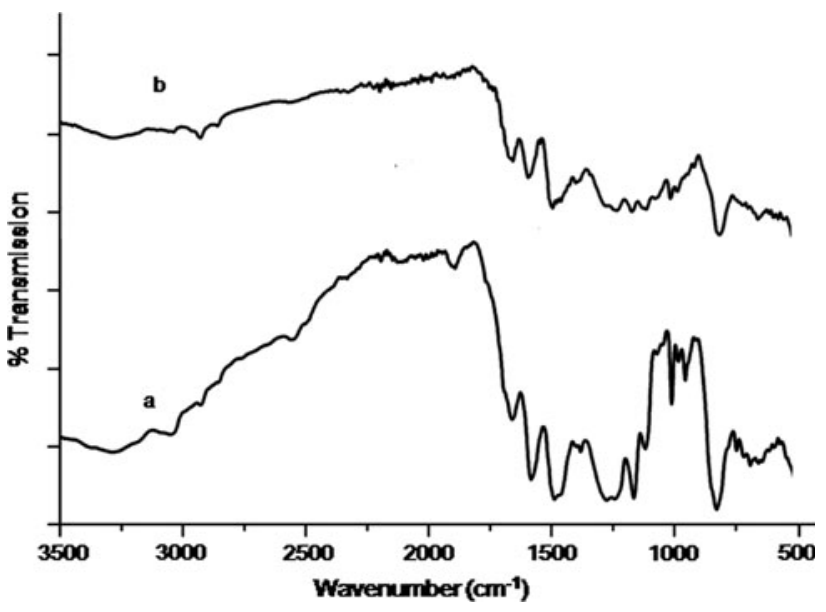

Fig. 3 Shows FTRI spectra of standard polyaniline (a) and PANiAgNPs nanocomposite $(b)$

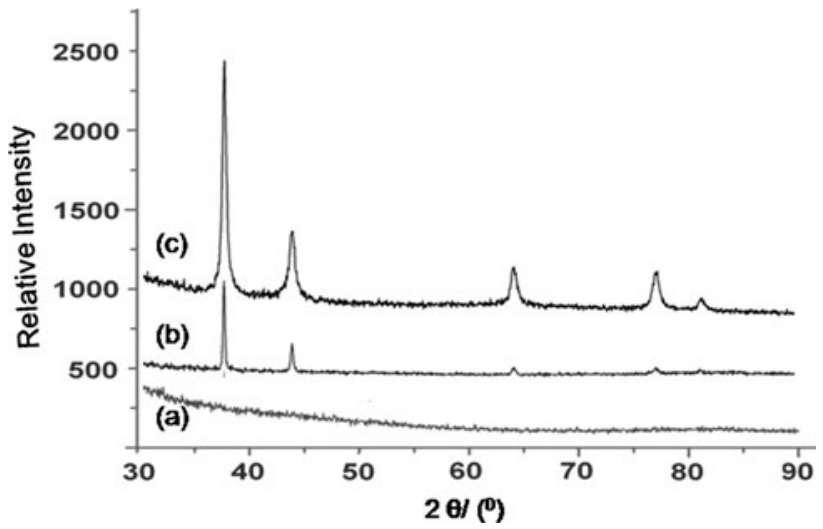

Fig. 4 Shows X-ray diffraction pattern of standard PANi (a) $1 \%$ AgNPs $(b)$ and PANi-AgNPs nanocomposite $(c)$ 
XRD pattern of polyaniline-silver nanocomposite shows characteristic peaks at $2 \theta=38,45,65,73$, and 77 representing Bragg's reflections from (111) (200) (220) (311) planes of the face centered cubic lattice phase (JCPDS no01-1164). Generally, the broadening of peaks in the X-ray diffraction pattern is related to smaller particle size when compared with (b), suggested the smaller AgNPs embedded in polyaniline.

Figure 5 shows the morphology of the polyaniline-silver nanocomposite It indicates that nano-sized PANi possess nanofibers morphology and influence strongly the composite morphology. Since, the silver nanoparticles were synthesized in the polyaniline solution, the nanoparticles embedded into the polyaniline matrix.

Figure 6 shows TGA of standard polyaniline (a) and polyaniline-silver nanocomposite (b) in the temperature range of $25-700{ }^{\circ} \mathrm{C}$. The thermogravimetric analysis indicates better thermal stability of the polyaniline-silver nanocomposite than that of standard polyaniline.

The ITO/PANi/AgNPs electrode was characterized by cyclic voltammetry, using a $0.1 \mathrm{~mol} \mathrm{~L}^{-1} \mathrm{H}_{2} \mathrm{SO}_{4}$ support electrolyte as shown in Fig. 6. The ITO/PANi/AgNPs electrode shows oxidation $(0.36 \mathrm{~V})$ and reduction $(0.12 \mathrm{~V})$ due to silver nanoparticles. The voltammograms correspond to a reversible system, at low scan rates i. e. $15 \mathrm{mV} \mathrm{s}^{-1}$. Upon varying the scan rate, the charge transfer was diffusion-limited up to $50 \mathrm{mV} \mathrm{s}^{-1}$, and shows linearity (data not shown). Furthermore, the ITO-PANi/AgNPs electrodes were stable, displaying practically the same voltammograms after four cycles in the presence of $0.1 \mathrm{M}$ phosphate buffer, pH 7.0 Fig. 7.

DPV technique have used for determination of hydrazine as shown in Fig. 8. The DPV gave a peak at $0.12 \mathrm{~V}$ for hydrazine in $0.1 \mathrm{M}$ PBS ( $\mathrm{pH}$ 7.0). The plot of the peak current versus concentration of hydrazine was shown in inset of Fig. 8 in the range of $0.0010-0.50 \mathrm{mM}$. The linear response obtained at low hydrazine concentrations have used to construct a hydrazine sensor.

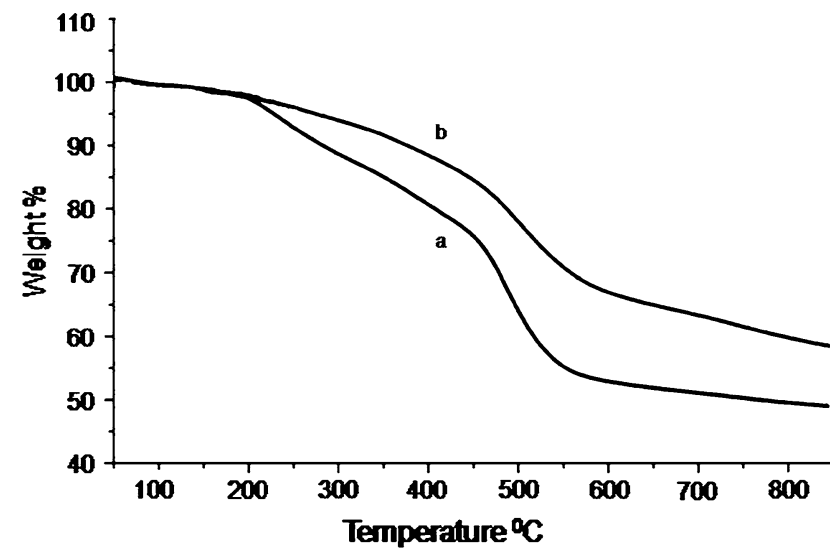

Fig. 6 Shows TGA of standard polyaniline (a) and polyaniline-silver nanocomposite $(b)$

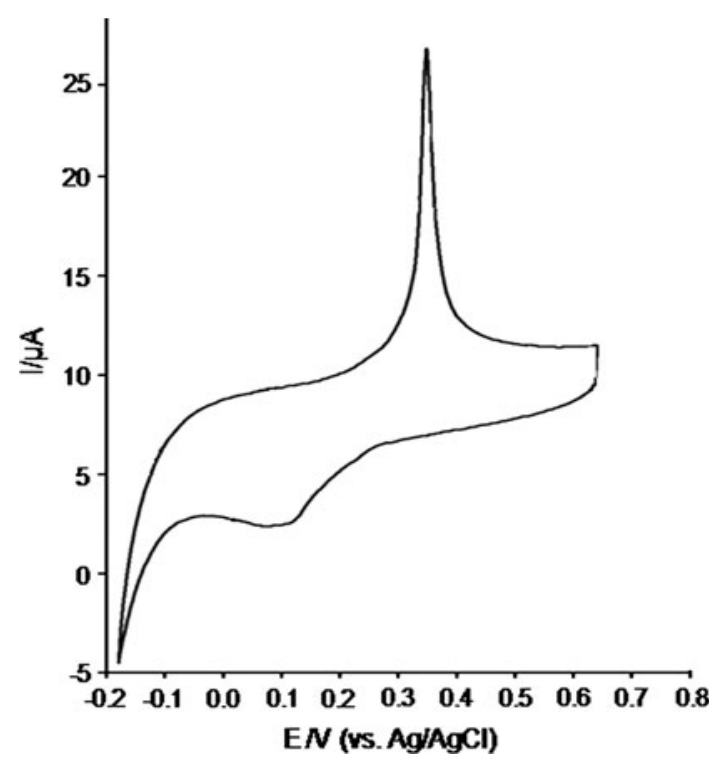

Fig. 7 Shows CV of ITO/PANi/AgNPs electrode using a $0.1 \mathrm{~mol} \mathrm{~L}^{-1}$ $\mathrm{H}_{2} \mathrm{SO}_{4}$ support electrolyte
Fig. 5 Shows SEM image of PANi-AgNPs nanocomposite
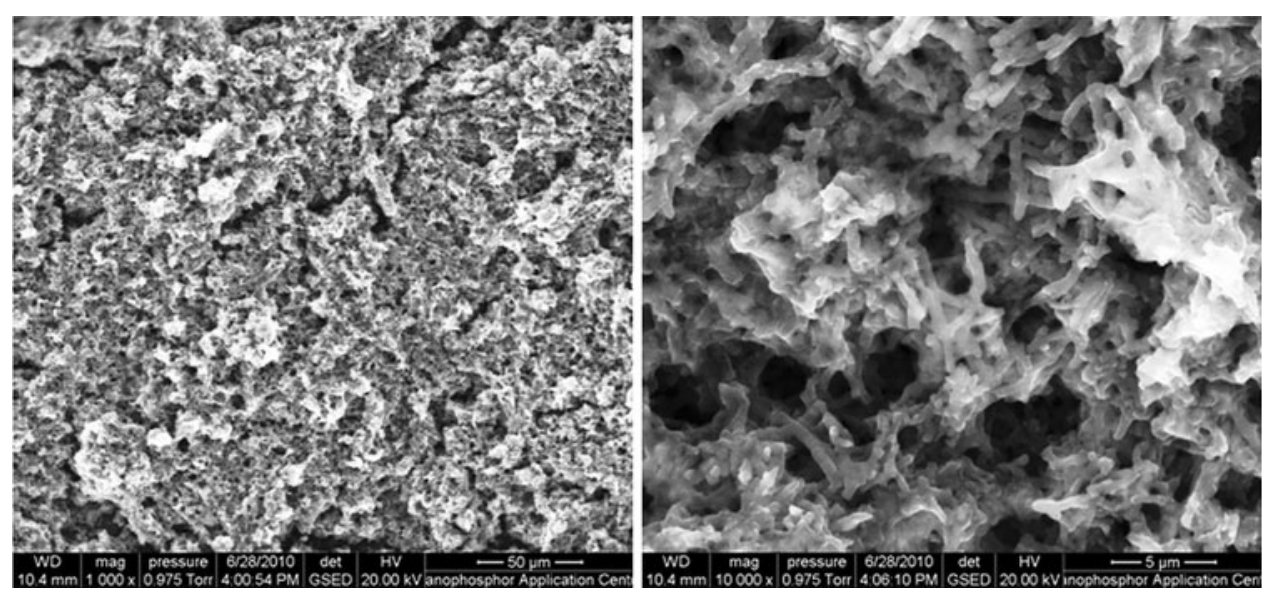


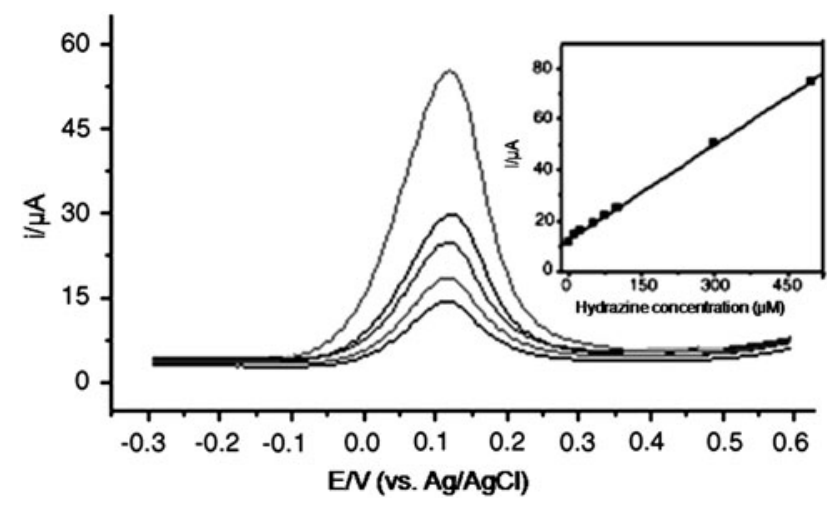

Fig. 8 Shows DPV for determination of hydrazine concentration in the range of $0.0010-0.50 \mathrm{mM}$ and inset shows the plot of peak current versus concentration of hydrazine

\section{Conclusions}

The synthesis of Ag-PANi nanocomposite by simple route is established in presence of aniline as a starting material. The photolysis is effective in the initial reduction of silver nitrate into AgNPs and then simulataneously act as photocatalyst to oxidize aniline to polyaniline. This nanocomposite is more thermally stable and more crystalline than standard polyaniline. Confirmation of the presence of silver nanoparticles in polyaniline is obtained by UV-Vis, PL, FTIR, XRD, TGA, SEM and CV analysis. The modified electrode are used for the first time to confirm the utility and application of this, towards the electrocatalytic oxidation of hydrazine.
Acknowledgment Work supported by DST Nano-Mission (SR/ NM/NS-87/2008), Department of Science and Technology.

Open Access This article is distributed under the terms of the Creative Commons Attribution Noncommercial License which permits any noncommercial use, distribution, and reproduction in any medium, provided the original author(s) and source are credited.

\section{References}

1. V.K. Shukla, R.P. Singh, A.C. Pandey, J. Alloy. Compds. 507, L13 (2010)

2. R.P. Singh, J.W. Choi, Adv. Mater. Lett. 1, 83 (2010)

3. R.P. Singh, A.C. Pandey, Anal. Methods 3, 586 (2011)

4. R.P. Singh, B.K. Oh, J.W. Choi, Sens. Transduc. J. 105, 104 (2009)

5. M. Vogel, A. Buldt, U. Karst, A critical review. Fresen. J. Anal. Chem. 366, 781 (2000)

6. E.D. Richter, A. Gal, E. Bitchatchi, A. Reches, Isr. J. Med. Sci. 28, 598 (1992)

7. P.K. Khanna, N. Singh, S. Charan, A.K. Viswanath, Mater. Chem. Phys. 92, 214 (2005)

8. Y.O. Kang, S.H. Choi, A. Gopalan, K.P. Lee, H.D. Kang, Y.S. Song, J. Non-Cryst. Solids 352, 463 (2006)

9. M.R. Karim, K.T. Lim, C.J. Lee, M.T.I. Bhuiyan, H.J. Kim, L.S. Park, M.S. Lee, J. Polym. Sci. A Polym. Chem. 45, 5741 (2007)

10. J. Du, Z. Liu, B. Han, Z. Li, J. Zhang, Y. Huang, Micropo.Mesoporo. Mater. 84, 254 (2005)

11. S.K. Pillalamarri, F.D. Blum, A.T. Tokuhiro, M.F. Bertino, Chem. Mater. 17, 5941 (2005)

12. H.H. Zhou, X.H. Ning, S.L. Li, J.H. Chen, Y.F. Kuang, Thin Solid Film 510, 164 (2006) 